\title{
Use of an attenuated live Salmonella Typhimurium vaccine on three breeding pig units: a longitudinal observational field study.
}

Davies, R. ${ }^{*}$, Gosling, R. J. ${ }^{*}$, Wales, A. D. ${ }^{*}$, Smith, R. P. ${ }^{\dagger}$

*Department of Bacteriology and Food Safety, and tDepartment of Epidemiological Sciences, Animal and Plant Health Agency (APHA - Weybridge), Woodham Lane, New Haw, Addlestone, Surrey, UK, KT15 3NB.

Author for correspondence: Andrew Wales

Email: research@andy.wales

Tel: $\quad+442920842402$ 


\section{Abstract}

The study examined the effects of a licensed live Salmonella Typhimurium vaccine, administered to sows and gilts on three commercial pig units experiencing clinical salmonellosis associated with S. Typhimurium or its monophasic variant. After vaccination, clinical salmonellosis resolved and shedding of $S$. Typhimurium declined markedly and persistently on all breeding or breedingfinishing units, during the one- to two-year monitoring period. On two finishing units supplied in part by one of the vaccinated herds, pigs from the vaccinated herd were less likely to shed Salmonella than those from non-vaccinating herds, and Salmonella counts in faeces were also lower from the vaccine-linked animals. Non-Typhimurium Salmonella serovars were isolated typically in fewer than $10 \%$ of samples, and showed no clear temporal changes in frequency. Vaccination of dams alone with $S$. Typhimurium was associated with reduced shedding of closely-related serovars among all age groups in this commercial setting.

Keywords: Salmonella, Typhimurium, vaccine, pig, swine
Abbreviations
APHA Animal and Plant Health Agency
BPW Buffered peptone water
CMI Cell-mediated immunity
mST Monophasic Salmonella Typhimurium
ST Salmonella Typhimurium 


\section{Introduction}

Salmonella in commercial pig production is a well-documented issue [1-3], with the principal concern in developed countries being one of public health risk from subclinical carriage on farms leading to contamination of food [4]. The control of Salmonella carriage and shedding by pigs, particularly in the finishing stages leading up to slaughter, can be challenging. The organism is robust in the environment, and infection cycles with currently-prevalent broad host range serovars such as Salmonella Typhimurium (ST) and its monophasic variant (mST) are frequently asymptomatic in livestock. Infection is readily acquired via oral or respiratory exposure, invasion of intestinal tissue and associated lymph nodes occurs, and shedding in faeces may be prolonged and/or reactivated by stress [5].

Strategies for control of Salmonella in pig herds in most countries typically attempt to reduce rather than eliminate infection or shedding, although aggressive policies involving segregation, depopulation, intensive cleaning and monitoring are practised successfully in some territories [6]. Controls to reduce Salmonella centre on biosecurity, reducing opportunities for new strains to enter the premises, and reducing infection pressure and transmission opportunities within a unit by means of hygiene, pest control, segregation and group management $[3,7,8]$. Animals' resistance to Salmonella infection can also be enhanced, in some cases by altering feed and feeding practices [9], but also by vaccination.

Over many years, candidate vaccines for Salmonella in pigs have developed, from killed bacterins injected parenterally to elicit a humoral response, to live or adjuvanted vaccines that additionally stimulate cell-mediated immunity (CMI). Humoral immunity alone has a limited effect on Salmonella [10], because for much of the infection cycle the organism is intracellular, shielded from antibody action. CMI, characterised by a Th1 lymphokine profile associated with activation of macrophages and cytotoxic lymphocytes, appears to be a critical part of effective anti-Salmonella 
immunity [11,12]. Live Salmonella vaccines, presented to mucosal surfaces, theoretically offer the best combination of antigen presentation and co-stimulatory signals to elicit CMI and mucosal immunity [13]. Live attenuated Salmonella strains offer the additional advantage for farmed species that inoculation can often be performed by non-parenteral routes (principally in drinking water or by aerosol), without the need for handling and injection of individual animals.

Whilst many experimental challenge studies have been reported involving candidate Salmonella vaccines $[13,14]$, relatively few have involved field studies, and still fewer have tackled field situations with broad host range serovars, such as are often seen in modern pig production. Among these, Arguello et al. [15] used an inactivated ST DT104 vaccine injected into grower pigs and demonstrated significant reductions in ST shedding after vaccination and in colonisation of mesenteric lymph nodes and caecum at slaughter, although Salmonella of other serogroups was not significantly reduced. Schwartz et al. [16] reported that a live Salmonella Choleraesuis (C1 serogroup) commercial vaccine, given orally to day-old piglets in an integrated production system with predominantly B-serogroup endemic strains, was associated with modest but significant decreases in individual-level seroprevalence and mesenteric lymph node colonisation at slaughter. Finally, a further trial used a different commercial $S$. Choleraesuis vaccine (cya/crp mutant) given in drinking water at three and 16 weeks of age to pigs on a unit with diverse endemic Salmonella serovars [17]. Compared with controls, there were significantly fewer Salmonella-positive ileocaecal lymph nodes among vaccinates at slaughter $(0.7 \%$ vs $7.2 \%)$.

'Salmoporc' is a live ST commercial vaccine strain, attenuated by mutagenesis to make it auxotrophic for adenine and histidine synthesis (Springer et al., 2001). Trials have reported safety and some degree of efficacy when it has been used in experimental ST-challenge studies. These have involved vaccinating dams and/or pre-weaned piglets [18], or vaccinating weaners subsequently challenged by oral dosing [19,20] or by seeder pigs [21]. The rationale to vaccinate 
dams is to reduce shedding around farrowing and to provide passive immunity to offspring [22].

Sometimes, the stimulus to adopt or trial a Salmonella vaccination programme is the appearance or persistence of a clinical salmonellosis problem on a unit. In the present observational studies, investigation of such problems on three breeding units associated with ST or its monophasic variant prompted trials of maternal vaccination using 'Salmoporc', with follow-up of the breeding and rearing animals by faeces and environmental sampling for up to two years after the initial (prevaccination) visit. Thus, the immediate aim of controlling clinical salmonellosis provided an opportunity for a more long-term assessment of the effects of vaccination on the various units upon subclinical shedding of Salmonella. This latter effect is potentially highly relevant to protection of consumers from Salmonella in the food chain.

\section{Materials and Methods}

\section{Farms}

Five UK pig farms were studied. The three breeding units (farms A1, B and C) were all experiencing clinical problems (diarrhoea, septicaemia, ill-thrift and increased mortality) in weaned pigs, associated with Salmonella infections.

Farm A1 was an outdoor breeding unit, recently stocked with around 800 gilts. Replacement gilts had been found to be heavily shedding ST and mST on arrival at around four weeks of age. The herd was moved to a different site between visits two and three. Farms A2 and A3 were outdoor grower/finisher units (part of a larger 20-site organisation) which received weaner pigs at around five weeks of age from Farm A1 and from other breeding farms that were not practising sow vaccination. One newly-established site was sampled on all occasions, whilst three sites of longer occupation were sampled once (two sites) or twice (one site). 
Farms B and C were large (400-sow) indoor breeder-finisher units. ST was present on Farm C and mST had been identified on Farm B, and problems with rodent control were evident on both these units.

\section{Vaccination}

Salmoporc STM (IDT Biologika, Dessau-Rosslau, Germany) was administered to pre-partum sows and gilts as two $(1 \mathrm{ml})$ subcutaneous injections, three weeks apart and two to three weeks before farrowing for the initial course, with a single booster dose two to three weeks before each subsequent farrowing.

\section{Sampling and Salmonella detection}

Multiple sampling visits were conducted, the first before the initial vaccinations and then on two to four additional occasions over the following year (Farms A2, A3, B and C) or two years (Farm A1). Sixty individual faeces samples were collected per epidemiological group (dry sows, lactating sows, weaners, etc.) where possible given the number of animals present, providing a $95 \%$ probability of detection given a $5 \%$ prevalence and $100 \%$ sensitivity of detection. Faeces were taken into sterile stool sample tubes using an integral spoon. In addition, pooled pen faeces samples (one or two pools per pen, including pre-weaned piglet faeces in farrowing accommodation) were taken, using a sterile gauze swab held with a clean disposable glove for each sample on indoor units or by making a pool from pinches of 10 droppings on outdoor units.

In addition, wildlife and environmental samples were collected. Solid and semi-solid material was collected using sterile gauze swabs, whilst surfaces were wiped with gauze swabs that had been preautoclaved in buffered peptone water (BPW). Materials and areas sampled included: rodent faeces and (occasionally) carcases; wild bird faeces; flies; cleaned and empty pens and farrowing crates; pooled water and mud in paddocks, wallows and tracks; water troughs; feed barrows and dust from 
feed handling areas; piglet transporters; handling facilities; manure heaps and associated runoff fluid; vehicles, trailers, loader buckets and scrapers. Details are given in the supplementary table.

Samples $(n=7201)$ were transported to the testing laboratory on the day of collection. Material was cultured for Salmonella either immediately upon arrival (pooled faeces and swabs in BPW) or after overnight storage at $4{ }^{\circ} \mathrm{C}$ (individual faeces samples), using a modification of the ISO 6579:2002 (Annex D) method, as described previously [23]. Briefly, all pooled faeces samples (approximately $25 \mathrm{~g}$ ) and swabs were pre-enriched in $225 \mathrm{ml} \mathrm{BPW}$ for $18 \mathrm{~h}$ followed by sequential inoculation onto Modified Semi-Solid Rappaport-Vassiliadis and Rambach agar. Sub-samples (2 g) of individual pig faeces samples and samples of aseptically dissected rodent carcass intestines plus liver and spleen, were pre-enriched in $20 \mathrm{ml} \mathrm{BPW}$ and cultured as above. The residue of the individual pig faeces samples were stored unprocessed at $4{ }^{\circ} \mathrm{C}$. Among individual faeces samples that proved Salmonella-positive, a representative subset of the stored material was subjected to a semiquantitative enumeration procedure by creating a decimal dilution series in BPW immediately before pre-enrichment, as described elsewhere [24]. A representative selection (all isolates from pooled samples and any individual sample that was cultured semi-quantitatively) of Salmonella isolates were fully sero- and phage-typed at the APHA Salmonella reference laboratory using standard methodology [25].

\section{Statistical analysis}

Statistical analyses were performed in Stata 12 (StataCorp, 2011. Stata Statistical Software: Release 12. College Station, TX: StataCorp LP). A mixed-effects logistic regression model was used to examine associations on farms A2 and A3 between finisher type (i.e. linked with vaccinated sows or not linked) and the odds of Salmonella-positive samples. Sample categories of environmental, individual faeces and pooled faeces were included a priori. Sampling visit number was included as a random effect, to account for the non-independence of samples collected at the same farm visit. 
For an overall analysis of the effect of vaccination on all of the farms, a mixed-effects logistic regression model was again used, to examine the association between time from the start of vaccination (represented by visit number, with the first visit being before the introduction of vaccination) and the odds of a sample being Salmonella-positive, the hypothesis being that vaccination would progressively reduce the odds of a sample being positive over time. The $a$ priori variables were sample (environmental, breeder, weaner, grower or finisher), faeces sample type (individual or pooled), and the number of days elapsed since the first visit to that farm. Farm identification was added as a random effect, with farms A1, A2 and A3 treated as a single farm in this respect. An interaction term was added to allow for different effects of the vaccine over visits on the different farms. The explanatory variable was the visit number, which was compared against two outcomes: Salmonella-positive or ST/mST-positive.

\section{Results}

On all farms clinical problems in weaners attributable to Salmonella, plus the need to use high doses of antibiotics at weaning, was reported by farmers and their veterinary advisers to be substantially improved after vaccination was introduced.

\section{Farm A1}

Five visits were made, between October 2010 and October 2012, with 422 to 605 samples collected per visit from the breeding herd. Weaners were not retained on the premises and consequently were not sampled. Bacteriological findings from faeces samples are summarised in Figure 1 and Table 1. The initial visit demonstrated a heavy prevalence of ST $/ \mathrm{mST}$ shedding $(45.3 \%$ of individual samples), with mST the more common. At the second visit, following the start of the vaccination programme, the reduction in prevalence of ST/mST-positive individual faeces samples was marked 
among ST/mST serovars. This comparatively low level of ST/mST shedding was evident at all subsequent visits, with $\mathrm{mST}$ always the more common variant. The reduction in the proportion of pooled samples positive for ST/mST was also marked, although more progressive over time than for the individual samples. Isolates of ST/mST from environmental and wildlife (mostly bird) samples similarly decreased progressively and markedly, from $62 \%$ of samples initially, to $11 \%$ and $1 \%$ on the last two visits. Rodent activity was subjectively assessed as low and localised, and the few rat faeces samples obtained were Salmonella-negative.

Non-ST/mST isolates from pigs were confined to serovars Panama and Derby, initially isolated only from pooled samples and at later visits from both individual and pooled samples. Environmental samples on occasion also yielded serovars Bovismorbificans, Newport and Reading. The most frequent isolations of non-ST/mST serovars in pooled faeces (17\%) and environmental (14\%) samples were made at the initial visit, which may have reflected largely transient shedding by the newly-arrived stock. On any visit thereafter, the proportion of samples positive for non-ST/mST Salmonella was between zero and $10 \%$ for any sample category (individual, pooled or environmental), with a trend of modest increase over time (Table 1). At the final visit, Salmonella Panama was the commonest serovar (including ST/mST) from all sample types.

\section{Farms A2 and A3}

Four visits were made, between November 2011 and December 2012, starting a year after the vaccination programme had commenced on Farm A1. Between 188 and 457 samples were taken on each occasion, sampling pigs from around six weeks of age and above. Findings from faeces samples are summarised in Figure 1 and Table 1. Shedding of ST/mST was not uniformly less frequent among linked pigs compared with unlinked pigs over the four visits. However, on visits one and two the semi-quantitative Salmonella counts of individual faeces samples among pigs linked to farm A1 (median and maximum log ranges 0-1 and 1-2 cfu.g ${ }^{-1}$, respectively) were lower 
than those from unlinked pigs (corresponding ranges 2-3 and 5-6 cfu. $\mathrm{g}^{-1}$ ). Heavy recent rainfall may have affected findings on the third and final visits, when the proportion of ST/mST-positive environmental samples increased to $67 \%$ and $44 \%$ respectively, having previously been around $10 \%$. Semi-quantitative counts on some individual faeces samples (median and maximum log ranges $0-1$ and $\leq 2-3$ on both occasions for both linked and unlinked pigs) indicated that shedding on these occasions, although more prevalent, was generally of low intensity.

None of the 1092 individual faeces samples from linked or unlinked pigs were positive for nonST/mST serovars. Nine of 16 sets of pooled or environmental samples were similarly negative. Of the other seven sets, between $2.1 \%$ and $8.3 \%$ of samples were positive for either Salmonella Derby or Salmonella Apapa.

\section{Farm B}

Three visits were made, between June 2012 and April 2013, and with 566-677 samples collected each time. By the time of the final visit, all finishing stock had been born to vaccinated dams. The unit staff reported a marked decline in clinical salmonellosis following the start of vaccination. Bacteriological findings are summarised in Figure 2 and Table 1. ST was not isolated. Monophasic ST was detected infrequently from individual faeces samples in the breeding herd. For this serovar the proportion of positive pooled samples was initially moderate $(30 \%)$ in this group, but this fell to below $1 \%$ on the two visits made after the start of the vaccination programme for breeding animals. Among weaners and growers, the prevalence of Salmonella isolations from individual samples fell rapidly after vaccination was introduced, from initially high values ( $>60 \%)$, to around or below $10 \%$ by the third visit. The proportion of positive pooled samples also fell, but more slowly and progressively. The initial prevalence of ST/mST-positive individual samples among the finisher pigs $(18.5 \%)$ was more modest than for the younger groups, but this too had reduced (to $1.7 \%$ ) by the third visit, with a parallel reduction in pooled sample isolations. Although the frequency of mST 
isolation from weaners' individual faeces samples reduced over time, Salmonella counts remained similar at each visit, with median and maximum log ranges of 1-2 and 3-5, respectively. However, among older growing stock initial maximum counts of 2-3 log units reduced to $0-1$ at subsequent visits.

A reduction among environmental sample isolations of ST/mST was also seen, from around $35 \%$ of samples on the first two visits to $6 \%$ on the last. Evidence of mouse activity was commonly seen (especially among growers) at the first visit, and attempts to improve control met with limited success. Few rodent faeces samples were obtained, as mice were living within the insulation layers of pig housing, but mST was commonly isolated from mouse faeces samples.

Non-ST/mST isolations were uncommon (less than $2 \%$ of samples per visit in any sampling category) and absent among weaners and growers. Most isolates were from the breeding herd and finishers, with serovars Anatum, Mbandaka and Stourbridge identified.

\section{Farm C}

Three visits were completed, between July 2011 and August 2012, with 396 to 422 samples being collected on each occasion from the breeding herd, weaners, and multi-age grower-finisher houses. The primary aim was to monitor the distribution of Salmonella across the farm by representatively sampling the animal groups present, and on Farm $\mathrm{C}$ the groups were particularly numerous and the pens diverse. Therefore, within funding constraints, a strategy was adopted of taking many pooled faeces samples but no individual ones. There was a marked decline in clinical salmonellosis after the start of vaccination, as reported by staff. Bacteriological findings are summarised in Figure 3 and Table 1. Monophasic ST was not isolated. The proportion of ST-positive pools reduced in all animal categories from initial pre-vaccination values, and in all cases the reduction was substantial by the third and final visit. Pooled faecal samples from finishing animals showed the least rapid reductions. A parallel reduction in environmental isolations of ST was also seen: $33 \%$ of samples 
initially, with $7.7 \%$ at the last visit. Rat control improved substantially over the study period but mice remained poorly controlled. In one finishing house ST in pooled samples did not decline as rapidly as in other houses, and mouse infestation here was noted to be especially severe.

Non-Typhimurium isolates were most commonly Salmonella Derby among breeding animals, and Salmonella Senftenberg among finishers and environmental samples. Serovars Havana, Cerro, Putten and Tennessee were also isolated from these last two sample categories. The frequency of isolation of non-Typhimurium Salmonella within animal categories varied from zero to $10.6 \%$ on any occasion but, unlike ST, there was no pattern of reducing isolations over time.

\section{Statistical analysis}

Findings from the logistic regression analyses are summarised in Table 2. Examining the relationship between maternal vaccination and sample Salmonella status among young-stock on farms $\mathrm{A} 2$ and $\mathrm{A} 3$, there was an increased odds ratio $(\mathrm{OR}=1.39)$ of a sample being positive among pigs not linked to vaccinated sows, although the difference did not achieve the conventional significance limit $(\mathrm{P}=0.076)$. A model that excluded data from the last two visits (when heavy rainfall may have affected the spread of Salmonella on these outdoor units) did show significantly increased odds $(\mathrm{P}<0.001)$ of Salmonella-positive samples among unlinked pigs, for all serovars $(\mathrm{OR}=4.21)$ and for $\mathrm{ST} / \mathrm{mST}$ alone $(\mathrm{OR}=3.85)$. The analysis of the frequency of Salmonella isolations over time on all the farms revealed significant reductions in the odds of isolation at all visits after the initial (pre-vaccination) visit, both for $S$. Typhimurium (or its monophasic variant) and for all serovars.

\section{Discussion}

The resolution of clinical salmonellosis on affected units following vaccination is consistent with 
other field research findings of same-serovar clinical protection by modified live Salmonella vaccines $[26,27]$. Furthermore, substantial and significant reductions in the proportion of ST/mSTpositive samples were seen on all breeding and breeding-finishing units following the start of the vaccination programmes.

On the finishing units (farms A2 and A3, receiving stock both from the vaccinating Farm A1 and non-vaccinating units elsewhere) the pattern of Salmonella isolation was more mixed than elsewhere. This may well have been influenced by heavy rainfall before the last two visits, with an increased prevalence but lower intensity of shedding (i.e. reduced Salmonella counts) being consistent with increased environmental challenge. Analysis for these units that excludes data from this latter period suggests an underlying effect of vaccination that is more marked than that shown by the complete dataset. Furthermore, the intensity of shedding on these two units appeared to be substantially reduced among offspring of vaccinated dams, compared with the other finishers. Similarly, a reduction in maximum Salmonella counts was observed among growers and finishers on Farm B after vaccination commenced.

These are several reasons to be careful about interpreting the present findings. These data arise from observational field studies, uncontrolled and non-randomised, where vaccination was prompted by the investigation of an existing Salmonella problem. Vaccination was undertaken in the context of other efforts to improve Salmonella control, variously by pest proofing and population control of rodents and wild birds. Therefore, some of the improvements in Salmonella shedding and contamination may potentially have been a consequence of these alternative measures. Furthermore, in some cases investigations and interventions may have been prompted because clinical salmonellosis and shedding were at a natural (and transient) peak. Therefore some improvement might have occurred owing to natural cycles of immunity within herds. There may also have been interactions, whereby (for example) vaccination may not have been effective if threshold levels of 
rodent control were not achieved. Finally, a proportion of the reduction seen for Farm A1 may have resulted from movement of the herd to new accommodation after the second visit.

Nonetheless, it is reasonable to postulate that the vaccination programmes at least contributed to the observed improvements in Salmonella control, and in many respects details in the data support this. Immediate and sustained improvements in ST and/or mST shedding were seen in all breeding groups after implementation of vaccination, even when rodent control was not markedly improved in some cases. The time lag seen with reductions in shedding by growing pigs on the breederfinisher units is also consistent with enhanced passive immunity and reduced carriage of infection by weaners, eventually propagating into growers and finishers. This would be the expected mechanism of maternal vaccination, if effective. The dramatic improvements among weaners and growers on Farm B are noteworthy as they suggest that, prior to vaccination, immunologically naïve weaners from a breeding herd with little active Salmonella infection were readily infected by mST either carried over from farrowing accommodation or within the nursery.

A slower reduction in Salmonella-positive pooled samples is consistent with a greater sensitivity for detection of shedding within a group for this sample type, compared with individual samples, as previously noted [28]. Thus, pooled samples appear not to be sensitive monitors of short-term responses to an intervention such as vaccination, but they are a sensitive monitor of residual Salmonella excretion, and indeed of an environmental reservoir for challenge such as in the breeding/farrowing environment in Farm B.

Growers and finishers exposed to fresh environmental Salmonella challenge, after passive immunity from vaccinated dams had waned would not be expected to show enhanced resistance to colonisation and shedding. In the situations where challenge to older growing stock was likely to be higher (farms A2 and A3 after heavy rain, Farm B house with heavy mouse infestation), there did 
indeed appear to be rather limited protection afforded to stock from vaccinated dams. Studies using Salmoporc and a ST challenge dose [18], or an autologous mST vaccine plus natural exposure [29], have reported that best protection against shedding and organ colonisation in growing pigs was seen when maternal plus juvenile Salmonella vaccination was performed in sequence. Nonetheless, where challenge levels after weaning can be kept low (by interventions that may include reduced carriage by passively-protected weaners), the present study suggests that maternal vaccination alone may provide protection that keeps infection pressure low throughout rearing. Compared with protocols involving juvenile vaccination, such a strategy can reduce administration costs and avoids the risk of vaccine strain being present in the lymph nodes of slaughtered pigs.

It is interesting in this context that the frequency of isolations of ST/mST from environmental sources tended to decline in parallel with that from the pooled faeces sample types. This suggests that environmental Salmonella levels on pig units largely reflect recent shedding by animals, rather than representing a primary driver for pig infection. A high environmental Salmonella level may be primary when there are specific external factors causing it, but even then the consequences for pig infection may vary according to whether the factor(s) result in spreading Salmonella (for example wet weather on an outdoor unit) or in focussing, intensifying and prolonging the challenge, such as may be seen with rodent infestations [30]. On farms A2 and A3, wet weather was followed by an increased frequency of shedding of Salmonella, but at comparatively low concentrations, indicating that this may have been transient cycling of environmental organisms without true colonisation of the gut.

It appeared that there was little or no control of non-mST/ST salmonellas following vaccination. An initial decline in other serovar isolations on Farm A1 probably reflects transient strains from the large numbers of brought-in stock, and in all cases either no pattern over time is evident, or there appears to have been a modest increase in alternative serovar isolations as $\mathrm{mST} / \mathrm{ST}$ declined. The 
significant reduction in isolation of 'all serovars' over time probably reflects the numerical dominance of $\mathrm{mST} / \mathrm{ST}$ isolations in the data. This pattern is consistent with the limitations of crossserovar protection against colonisation and shedding seen in experimental studies [31-34]. Few of the non-mST/ST serovars isolated were commonly isolated from UK wildlife in the same period [35], thus indicating that feed and/or incoming stock were the likely sources. Many of these alternative serovars are likely to have been transient, shedding frequency was generally low, and none of them carry a similarly high pathogenic importance for humans as ST or mST.

With the current circumstances, in Europe and elsewhere, of herd Salmonella infections typically being subclinical and associated with broad-host-range serovars [1,36], the use of vaccination is increasingly focussed on the reduction of Salmonella risk at slaughter and onward into the human food chain, without interfering with monitoring schemes. If vaccination is applied at an early stage in production (and particularly ante-partum), the risk of adverse effects on monitoring for Salmonella at slaughter by serology or culture is reduced. With modern biotechnologies and immunological insights, Salmonella vaccines show considerable promise for making a substantial contribution to the safety of food from the pig sector [37]. However, longitudinal field studies (such as the present one) examining natural infections are comparatively uncommon amongst reports of Salmonella vaccination trials in pigs, despite such situations being the target of most commercial applications of vaccination.

The current study provides promising evidence of control of salmonellosis and sustained reductions in ST and mST shedding among pigs up to slaughter age, following vaccination of dams with a live attenuated ST vaccine. Observed alterations in shedding differed between pig units, and other factors may have contributed to the effects observed. Controlled interventional field trials would be needed to quantify the respective contributions of these various factors. 


\section{Acknowledgements}

The authors would like to thank the farmers involved in the present study. The work was funded by the UK Department for Environment, Food and Rural Affairs (Defra) under projects FZ2015, OZO342 and OZO344.

\section{Declaration of interests}

The authors declare no conflicts of interest. 


\section{References}

[1] EFSA, Report of the task force on zoonoses data collection on the analysis of the baseline survey on the prevalence of Salmonella in slaughter pigs, Part A: Salmonella prevalence estimates, EFSA J. 6 (2008) 111 pp. doi:10.2903/j.efsa.2008.135r.

[2] Y.-H. Kim, I.-K. Kwon, J.-H. Han, Seroprevalence of swine salmonellosis in Korean swine herds, Korean J. Food Sci. Anim. Resour. 30 (2010) 62-65.

[3] M.H. Rostagno, T.R. Callaway, Pre-harvest risk factors for Salmonella enterica in pork production, Food Res. Int. 45 (2012) 634-640. doi:10.1016/j.foodres.2011.04.041.

[4] T. Hald, D. Vose, H.C. Wegener, T. Koupeev, A Bayesian approach to quantify the contribution of animal-food sources to human salmonellosis, Risk Anal. 24 (2004) 255-69.

[5] F. Boyen, F. Haesebrouck, D. Maes, F. Van Immerseel, R. Ducatelle, F. Pasmans, Nontyphoidal Salmonella infections in pigs: A closer look at epidemiology, pathogenesis and control, Vet. Microbiol. 130 (2008) 1-19. doi:10.1016/j.vetmic.2007.12.017.

[6] P. Hopp, H. Wahlstrom, J. Hirn, A common Salmonella control programme in Finland, Norway and Sweden, Acta Vet. Scand. Supplementum 91 (1999) 45-9.

[7] M.E.E. Ball, E. Magowan, M. Taylor, G. Bagdonaite, R. Madden, A review of current knowledge on Salmonella control on-farm and within the processing plant relevant to the Northern Ireland pig industry, Agri-food and Biosciences Institute (AFBI), Belfast, UK, 2011. http://www.afbini.gov.uk/salmonella-lit-review-feb-2011.pdf.

[8] S. Belluco, V. Cibin, R. Davies, A. Ricci, A. Wales, A review of the scientific literature on the control of Salmonella spp. in food producing animals other than poultry (OIE thematic publication), World Organisation for Animal Health (OIE), 2015. http://web.oie.int/boutique/.

[9] M.S. Hedemann, L.L. Mikkelsen, P.J. Naughton, B.B. Jensen, Effect of feed particle size and feed processing on morphological characteristics in the small and large intestine of pigs and on adhesion of Salmonella enterica serovar Typhimurium DT12 in the ileum in vitro, J. Anim. Sci. 83 (2005) 1554-1562.

https://www.animalsciencepublications.org/publications/jas/articles/83/7/0831554.

[10] J.D.P. O’Brien, An outbreak of Salmonella cholerae-suis infection in a large pig fattening enterprise in Lancashire, Vet. Rec. 79 (1966) 558-561.

[11] A.A. Lindberg, J.A. Robertsson, Salmonella typhimurium infection in calves: cell-mediated and humoral immune reactions before and after challenge with live virulent bacteria in calves given live or inactivated vaccines, Infect. Immun. 41 (1983) 751-757. http://iai.asm.org/cgi/content/abstract/41/2/751.

[12] M.P. Murtaugh, Advances in swine immunology help move vaccine technology forward, Vet. Immunol. Immunopathol. 159 (2014) 202-207. doi:10.1016/j.vetimm.2014.02.017.

[13] F. Haesebrouck, F. Pasmans, K. Chiers, D. Maes, R. Ducatelle, A. Decostere, Efficacy of vaccines against bacterial diseases in swine: what can we expect?, Vet. Microbiol. 100 (2004) 255-268. doi:10.1016/j.vetmic.2004.03.002.

[14] P.A. Barrow, U. Methner, Vaccination against Salmonella infections in food animals: rationale, theoretical basis and practical application, in: P.A. Barrow, U. Methner (Eds.), Salmonella Domest. Anim., 2nd ed., CAB International, Wallingford, UK, 2013: pp. 455-475.

[15] H. Arguello, A. Carvajal, G. Naharro, P. Rubio, Evaluation of protection conferred by a Salmonella Typhimurium inactivated vaccine in Salmonella-infected finishing pig farms, Comp. Immunol. Microbiol. Infect. Dis. 36 (2013) $489-498$. doi:10.1016/j.cimid.2013.05.002.

[16] P. Schwarz, J.D. Kich, J. Kolb, M. Cardoso, Use of an avirulent live Salmonella Choleraesuis vaccine to reduce the prevalence of Salmonella carrier pigs at slaughter, Vet. Rec. 169 (2011) 553. doi:10.1136/vr.d5510.

[17] D. Maes, K. Gibson, E. Trigo, A. Saszak, J. Grass, A. Carlson, et al., Evaluation of crossprotection afforded by a Salmonella Choleraesuis vaccine against Salmonella infections in pigs under field conditions, Berl. Münch. Tierärztl. Wochenschr. 114 (2001) 339-341. 
[18] U. Roesler, M. Stief, M. Leffler, U. Truyen, J. Lehmann, I. Szabo, et al., Persistence, excretion and efficacy of an attenuated Salmonella vaccine in suckling piglets, Prakt. Tierarzt. 91 (2010) $59-64$.

[19] M. Selke, J. Meens, S. Springer, R. Frank, G.-F. Gerlach, Immunization of pigs to prevent disease in humans: Construction and protective efficacy of a Salmonella enterica serovar Typhimurium live negative-marker vaccine, Infect. Immun. 75 (2007) 2476 -2483. doi:10.1128/IAI.01908-06.

[20] S. Springer, T. Lindner, G. Steinbach, H.J. Selbitz, Investigation of the efficacy of a genetically-stabile live Salmonella Typhimurium vaccine for use in swine, Berl. Münch. Tierärztl. Wochenschr. 114 (2001) 342 -345.

[21] L. De Ridder, D. Maes, J. Dewulf, F. Pasmans, F. Boyen, F. Haesebrouck, et al., Evaluation of three intervention strategies to reduce the transmission of Salmonella Typhimurium in pigs, Vet. J. 197 (2013) 613 -618. doi:10.1016/j.tvj1.2013.03.026.

[22] A.D. Wales, A.J.C. Cook, R.H. Davies, Producing Salmonella-free pigs: a review focusing on interventions at weaning, Vet. Rec. 168 (2011) 267-276. doi:10.1136/vr.d1125.

[23] F. Martelli, R. Gosling, I. McLaren, A. Wales, R. Davies, Development and testing of external quality assessment samples for Salmonella detection in poultry samples, Lett. Appl. Microbiol. 59 (2014) 443-448. doi:10.1111/lam.12299.

[24] A. Wales, M. Breslin, R. Davies, Semiquantitative assessment of the distribution of Salmonella in the environment of caged layer flocks, J. Appl. Microbiol. 101 (2006) 309-318. doi:10.1111/j.1365-2672.2006.02916.x.

[25] Y.E. Jones, I.M. McLaren, C. Wray, Laboratory aspects of Salmonella, in: C. Wray, A. Wray (Eds.), Salmonella Domest. Anim., 1st ed., CABI Publishing, Wallingford, UK, 2000: pp. 393405.

[26] T.T. Kramer, M.B. Roof, R.R. Matheson, Safety and efficacy of an attenuated strain of Salmonella choleraesuis for vaccination of swine, Am. J. Vet. Res. 53 (1992) $444-8$.

[27] H.W. Smith, The immunization of mice, calves and pigs against Salmonella dublin and Salmonella cholerae-suis infections, J. Hyg. (Lond.). 63 (1965) 117-135. http://www.ncbi.nlm.nih.gov/pmc/articles/PMC2134622/.

[28] M.E. Arnold, A.J.C. Cook, Estimation of sample sizes for pooled faecal sampling for detection of Salmonella in pigs, Epidemiol. Infect. 137 (2009) 1734-1741. doi:10.1017/S0950268809002702.

[29] J. Ruggeri, M. Pesciaroli, F. Foresti, E. Giacomini, M. Lazzaro, M.C. Ossiprandi, et al., Inactivated Salmonella enterica serovar Typhimurium monophasic variant $(S$. Typhimurium $\left.1,4,[5], 12: i_{-}\right)$in sows is effective to control infection in piglets under field condition, Vet. Microbiol. 180 (2015) 82-89. doi:10.1016/j.vetmic.2015.07.029.

[30] R.H. Davies, A.D. Wales, Salmonella contamination of cereal ingredients for animal feeds, Vet. Microbiol. 166 (2013) 543-9. http://dx.doi.org/10.1016/j.vetmic.2013.07.003.

[31] S.D. Charles, A.S. Abraham, E.T. Trigo, G.F. Jones, T.L. Settje, Reduced shedding and clinical signs of Salmonella Typhimurium in nursery pigs vaccinated with a Salmonella choleraesuis vaccine, Swine Health Prod. 8 (2000) $107-112$.

[32] D.L. Foss, T.S. Agin, D. Bade, D.A. Dearwester, R. Jolie, R.L. Keich, et al., Protective immunity to Salmonella enterica is partially serogroup specific, Vet. Immunol. Immunopathol. 155 (2013) 76 -86. doi:10.1016/j.vetimm.2013.06.007.

[33] K.J. Groninga, E. Springer, M. Braunschmidt, D. Pankratz, Salmonella Derby crossprotection study, Vet. Ther. 1 (2000) 59-62. https://www.vetlearn.com/veterinarytherapeutics/salmonella-derby-cross-protection-study.

[34] J.A. Husa, R.A. Edler, D.H. Walter, J.T. Holck, R.J. Saltzman, A comparison of the safety, cross-protection, and serologic response associated with two commercial oral Salmonella vaccines in swine, J. Swine Health Prod. 17 (2009) 10-21. https://www.aasv.org/shap/issues/v17n1/v17n1p10.htm.

[35] AHVLA, Salmonella in Livestock Production in GB 2013, Animal Health and Veterinary 
Laboratories Agency, UK, 2014. https://www.gov.uk/government/statistics/salmonella-inlivestock-production-in-great-britain-2013.

[36] A. Farzan, R.M. Friendship, C.E. Dewey, C. Poppe, J. Funk, C.A. Muckle, A longitudinal study of the Salmonella status on Ontario swine farms within the time period 2001-2006, Foodborne Pathog. Dis. 5 (2008) 579-588. doi:10.1089/fpd.2007.0074.

[37] A.D. Wales, R.H. Davies, Salmonella vaccination in pigs: a review, Zoonoses Public Health. In press (2016). doi:10.1111/zph.12256. 
Table 1: Isolations of Salmonella from individual and naturally-pooled faeces plus environmental samples on the five study farms

\begin{tabular}{|c|c|c|c|c|c|c|c|c|c|}
\hline \multirow{3}{*}{$\begin{array}{l}\text { Farm, } \\
\text { date of } \\
\text { visit }\end{array}$} & \multicolumn{9}{|c|}{ Percentage of Salmonella-positive samples: Typhimuriuma, non-Typhimurium (total number of samples) } \\
\hline & \multicolumn{2}{|l|}{ Breeding herd } & \multicolumn{2}{|l|}{ Weaners } & \multicolumn{2}{|c|}{$\begin{array}{l}\text { 'Unlinked' animals (Farms A2 \& } \\
\text { A3) })^{\mathrm{b}} \text {, or Growers (B) }\end{array}$} & \multicolumn{2}{|c|}{$\begin{array}{l}\text { 'Linked' animals (Farms A2 \& A3) })^{\mathrm{b}} \text {, } \\
\text { Finishers (B), Growers/Finishers (C) }\end{array}$} & \multirow{2}{*}{$\begin{array}{l}\text { Environmental/ } \\
\text { wildlife samples }\end{array}$} \\
\hline & Individual & Pooled & Individual & Pooled & Individual & Pooled & Individual & Pooled & \\
\hline \multicolumn{10}{|l|}{ A1 } \\
\hline Oct '10 & $\underline{45.3,} \quad 0.0(375)$ & $\underline{73.9,17.4(23)}$ & & & & & & & $61.9,14.3(63)$ \\
\hline Mar '11 & 7.4, $0.4(282)$ & 25.0, $0.0(56)$ & & & & & & & 28.6, $0.0(84)$ \\
\hline Oct '11 & $2.0, \quad 0.3(344)$ & 7.7, $1.9(52)$ & & & & & & & $\mathbf{5 . 1}, 4.0,(198)$ \\
\hline Apr'12 & $5.2,2.6(346)$ & $8.9, \quad 7.1 \quad(56)$ & & & & & & & $11.3,5.9,(203)$ \\
\hline Oct '12 & $1.3,4.9(305)$ & $2.9, \quad 3.6(137)$ & & & & & & & $\mathbf{0 . 8}, 9.8,(133)$ \\
\hline \multicolumn{10}{|l|}{$A 2 \& A 3$} \\
\hline Nov '11 & & & & & $7.80 .0(180)$ & $42.9,7.1$ (14) & $2.5, \quad 0.0(120)$ & $8.8, \quad 2.9 \quad(34)$ & $12.8,2.1 \quad(47)$ \\
\hline May '12 & & & & & $2.20 .0(186)$ & $19.4,2.8$ (36) & $09,0.0(116)$ & $8.3,0.0 \quad(24)$ & $10.0,5.0 \quad(20)$ \\
\hline Jul ‘12 & & & & & $4.60 .0(216)$ & $38.9,8.3 \quad(36)$ & $11.1, \quad 0.0(144)$ & 50.0, $0.0 \quad(24)$ & $67.6, \quad 0.0 \quad(37)$ \\
\hline Dec '12 & & & & & $28.1 \quad 0.0 \quad(32)$ & $50.0, \quad 0.0 \quad(6)$ & $13.9, \quad 0.0 \quad(96)$ & $38.5, \quad 0.0 \quad(18)$ & $44.4, \quad 2.8 \quad(36)$ \\
\hline \multicolumn{10}{|l|}{ B } \\
\hline Jun '12 & $3.7, \quad 0.0(163)$ & $30.2,2.6(116)$ & $\underline{61.7,} \quad 0.0 \quad(60)$ & 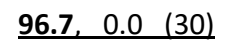 & $\underline{66.7,} \quad 0.0 \quad(24)$ & $84.6,0.0 \quad(26)$ & $\underline{18.5,} 0.0 \quad(81)$ & $\underline{60.0,} 3.1 \quad(65)$ & $33.3,0.0 \quad$ (3) \\
\hline Dec '12 & $0.0, \quad 0.7(150)$ & $\mathbf{0 . 8}, \quad 0.0(123)$ & $18.3,0.0(60)$ & $58.3, \quad 0.0 \quad(48)$ & $\begin{array}{lll}5.0, & 0.0 & (60)\end{array}$ & $\begin{array}{lll}55.6 & 0.0 \quad(18)\end{array}$ & $\begin{array}{lll}5.6, & 0.0 & (90)\end{array}$ & $66.7,1.2(81)$ & $34.5, \quad 0.0 \quad(29)$ \\
\hline Apr '13 & 0.6, $0.6(179)$ & $0.9, \quad 1.8(112)$ & $11.7,0.0 \quad(60)$ & $29.8, \quad 0.0 \quad(57)$ & $5.0, \quad 0.0 \quad(60)$ & $33.3,0.0 \quad(18)$ & $1.7, \quad 0.0(60)$ & 15.9, $1.6(63)$ & $5.9,1.5 \quad(68)$ \\
\hline \multicolumn{10}{|l|}{ C } \\
\hline Jul '11 & & $8.5,0.7(142)^{c}$ & & $\underline{22.2,} \quad 0.0 \quad(99)$ & & & & $19.4,7.5 \quad(93)$ & $33.3,4.5 \quad(66)$ \\
\hline Jan '12 & & $0.0, \quad 5.3(131)^{c}$ & & $0.9, \quad 0.0(108)$ & & & & 17.0, 2.3 (88) & $0.0, \quad 2.9 \quad(69)$ \\
\hline Aug '12 & & $\mathbf{0 . 8}, 0.8(122)^{\mathrm{c}}$ & & $\mathbf{0 . 0}, \quad 0.0(122)$ & & & & $4.4,10.6(113)$ & 7.7, $7.7 \quad(65)$ \\
\hline
\end{tabular}

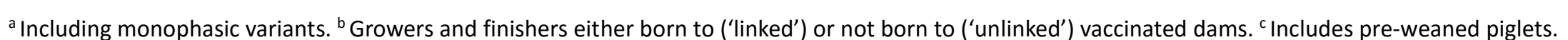

Underlined data is from before the start of the vaccination programmes 
Table 2: Results of logistic regression analyses on Salmonella status of faecal and environmental swab samples, from farms accommodating Salmonella-vaccinated dams and their offspring

\begin{tabular}{|c|c|c|c|c|c|}
\hline & \multirow{2}{*}{$\begin{array}{l}\text { No. of days after pre- } \\
\text { vaccination sampling }\end{array}$} & \multicolumn{2}{|c|}{ Salmonella-positive } & \multicolumn{2}{|c|}{ ST / mST-positive } \\
\hline & & Odds ratio & $p$-value & Odds ratio & $p$-value \\
\hline \multicolumn{6}{|l|}{ Farms A2/A3 } \\
\hline Linked & - & Ref. & - & Ref. & - \\
\hline Unlinked & - & 1.39 & 0.076 & 1.25 & 0.232 \\
\hline \multicolumn{6}{|l|}{ All farms } \\
\hline Visit 1 & 0 & Ref. & - & Ref. & - \\
\hline Visit 2 & $161-182$ & 0.033 & $<0.001$ & 0.01416 & $<0.001$ \\
\hline Visit 3 & $308-402$ & 0.002 & $<0.001$ & 0.00029 & $<0.001$ \\
\hline Visit 4 & $541-569$ & 0.002 & $<0.001$ & 0.00008 & $<0.001$ \\
\hline Visit 5 & 640 & 0.002 & 0.001 & 0.00008 & $<0.001$ \\
\hline Visit 6 & $729-792$ & 0.001 & 0.001 & 0.00001 & $<0.001$ \\
\hline
\end{tabular}

Results from farms A2/A3 compare Salmonella status of samples from pigs born to vaccinated dams ('linked') with those from premises where vaccination was not performed ('unlinked'); $n=1422$. Results from 'all farms' compare Salmonella status over time, compared with an initial prevaccination baseline; $n=6416$. Not all farms had more than three visits and 'visit number' in the analysis indicates the time band within which the visit occurred; in some cases this does not correlate strictly with the cumulative number of visits to a farm.

a Salmonella Typhimurium / monophasic S. Typhimurium. 


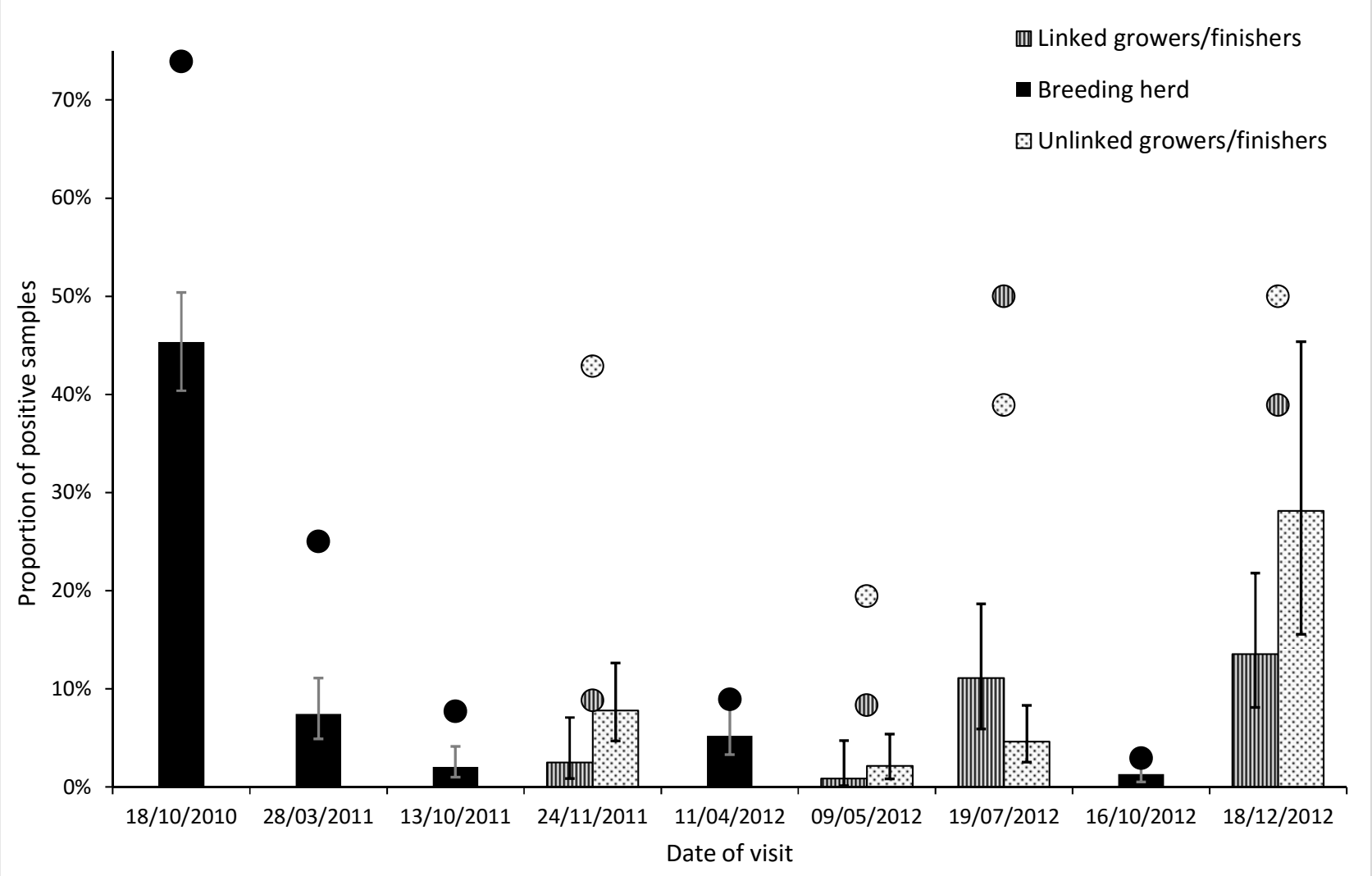

Bars show isolations from individual faeces samples, whilst circles show isolations from naturally-pooled faeces samples. 'Linked' animals were born to Salmonella-vaccinated dams, whilst 'unlinked' ones were not. Error bars show 95\% confidence intervals for the individual sample prevalence data. Salmonella vaccination of sows on Farm A commenced between the first and second sampling occasions.

Figure 1: Isolations of $S$. Typhimurium and monophasic $S$. Typhimurium from Farm A1 (black) and farms A2 and A3 (patterned) over a two-year period. 


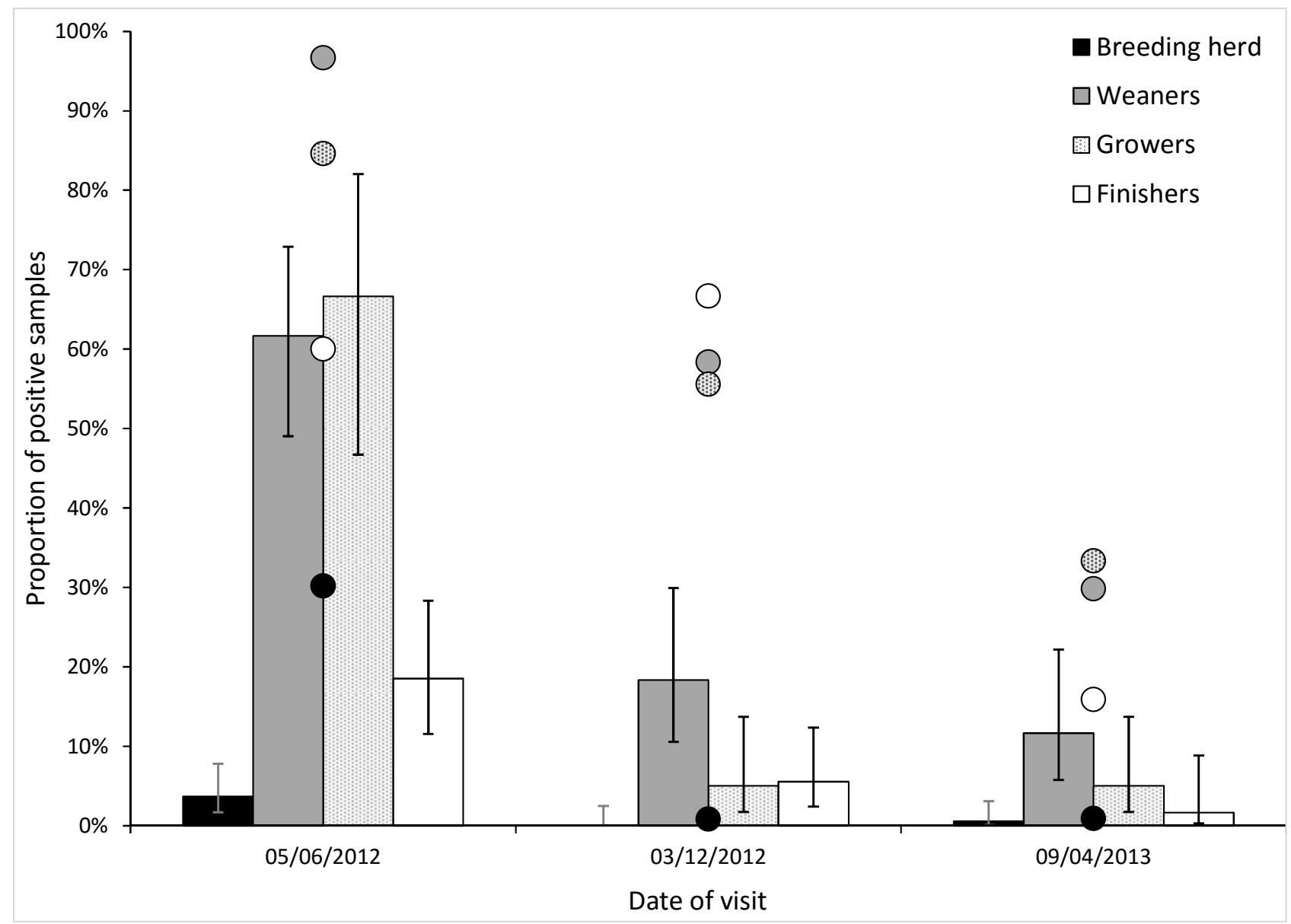

Bars show isolations from individual faeces samples, whilst circles show isolations from naturally-pooled faeces samples. Error bars show 95\% confidence intervals for the individual sample prevalence data. Salmonella vaccination of sows commenced between the first and second sampling occasions.

Figure 2: Isolations of monophasic S. Typhimurium from Farm B 


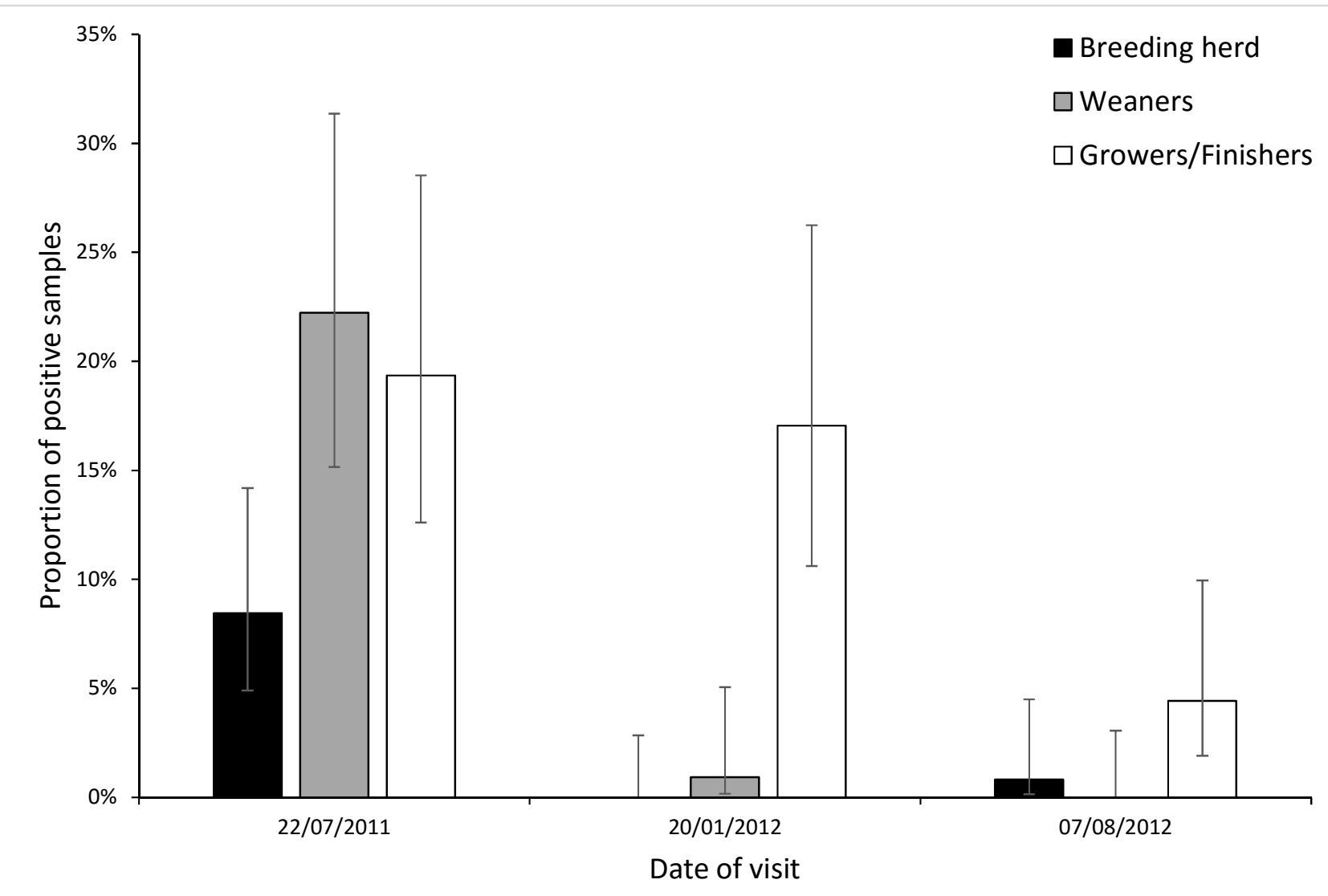

Isolations from naturally-pooled faeces samples. Error bars show $95 \%$ confidence intervals. Salmonella vaccination of sows commenced between the first and second sampling occasions.

Figure 3: Isolations of S. Typhimurium from Farm C 